\title{
Pb Isotopes Data from the Campanian Volcanic Province: A Model to Generate These Distinctive Pb Isotopic Variations
}

\author{
Angelo Paone ${ }^{1,2}$ \\ ${ }^{1}$ United States Geological Survay, Reston, USA \\ 2Dipartimento di Scienze della Terra, Università degli Studi di Napoli “Federico II", Naples, Italy \\ Email: angelo.paone1@gmail.com
}

Received 20 December 2014; accepted 11 January 2015; published 20 January 2015

Copyright @ 2015 by author and Scientific Research Publishing Inc.

This work is licensed under the Creative Commons Attribution International License (CC BY). http://creativecommons.org/licenses/by/4.0/

(c) (i) Open Access

\begin{abstract}
A broad set of samples from the CVP has been analyzing Pb isotopes. Campanian Volcanic Province (CVP) has been produced by various ranges of mixing between three components: 1) MORB, 2) Hercynian basement and 3) upper crust. Anyhow, the upper crust that has modified the $\mathrm{Pb}$ isotopic composition also shows trends towards an Achaean upper crust. This is consistent for all Mediterranean area. This is not in contrast with the past plate tectonic reconstruction. Also the surface sediments (GLOSS) may have been a mixing between the Achaean upper crust and a Hercynian component with an implication worldwide due to the rock cycle processes.
\end{abstract}

\section{Keywords}

Pb Isotopes, Campanian Volcanic Province, Hercynian, Sediments, MORB

\section{Introduction}

The Campanian Volcanic Province (Figure 1) is made of the following volcanic area: Somma-Vesuvius volcano [1], Campi Flegrei Volcanic District [2] [3], Roccamonfina volcano [3] and the Island of Ponza and Ventotene [4]. A tectonic scenario has been envisaged on the basis of geophysical, petrologic and geochemical data, where the magma is stored in the deep and hot intrusion zone [4]-[6]. The magmas erupted have a sediments signature due to the adjacent Adria slab subducting [4] [7]. Tomographic results confirm this scenario ([8], references therein). In this report, I would like to unravel the features of the $\mathrm{Pb}$ isotopes data from the Campanian Volcanic Province [9]-[11]. Moreover, a model is built to explain these typical $\mathrm{Pb}$ isotopes signatures. 


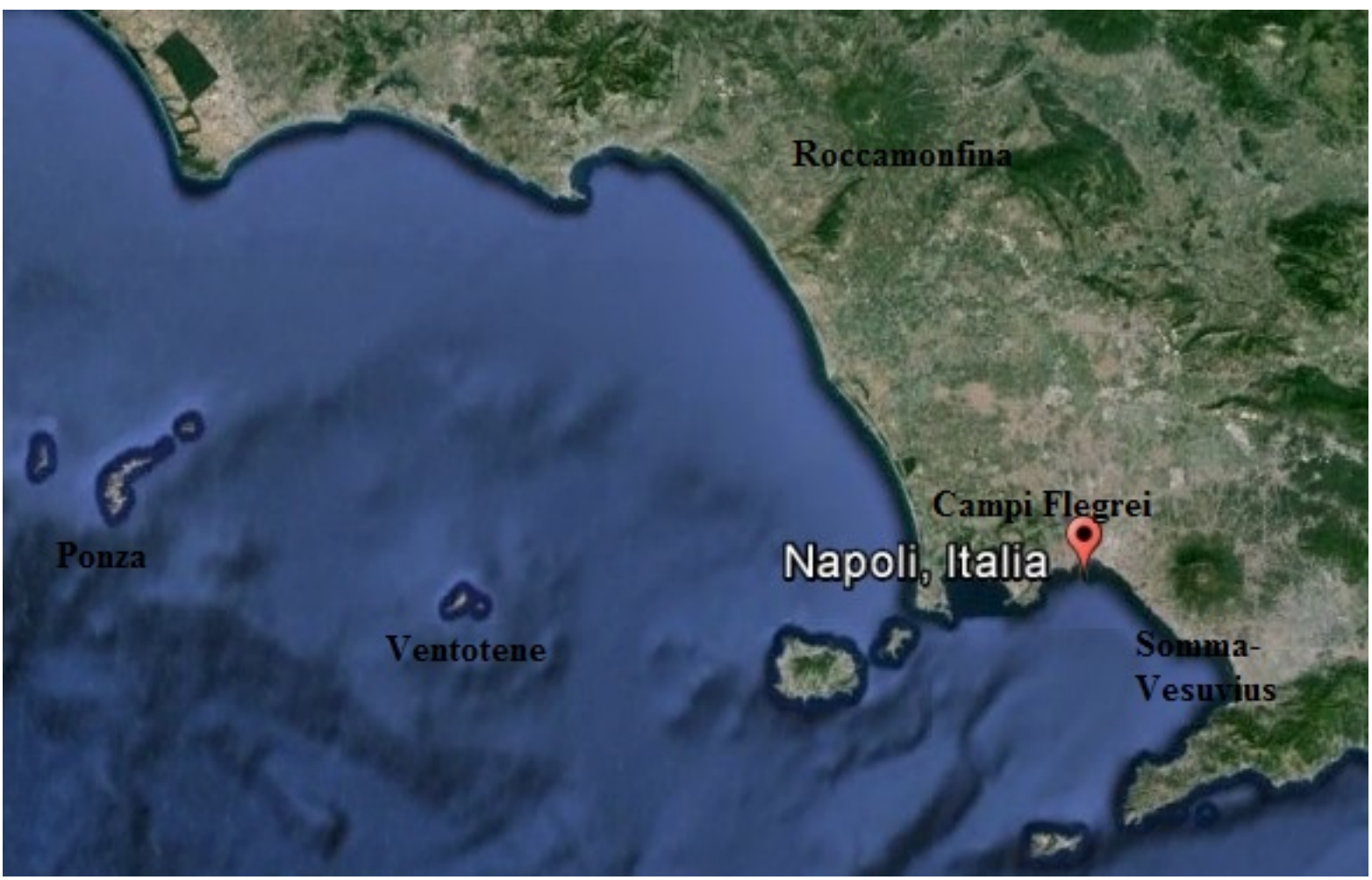

Figure 1. Aerial photograph of the main volcanic area of the Campanian Volcanic Province.

\section{A Geochemical Overview to Summarize the Magmatic Origin and Crustal Effects in Campanian Volcanic Province}

The Campanian Volcanic Province is part of the classic western potassic volcanic province of the Italian Peninsula. The Campanian volcanic products show the effects of shallow assimilation and fractional crystallization, and the contribution of regional crustal sources (e.g., Hercynian basement-Calabrian crust). The Roccamonfina, Campi Flegrei, and Ventotene volcanic rocks are characterised by wide isotopic and geochemical variations. Such variations appear to reflect both AFC processes and chemical heterogeneity in the upper mantle that may be linked to subduction processes. Mixing curves (Th/Ce-, Ba/K- and $\mathrm{Eu} / \mathrm{Eu}^{*}{ }_{-}{ }^{143} \mathrm{Nd} /{ }^{144} \mathrm{Nd}$ ) linking sediments and mantle end-members account for the variations in the Campanian Province volcanic rocks with a sediment contribution of $2 \%$ - $10 \%$. The upper mantle sources for the low- and high-K rocks at Roccamonfina have been constrained on the basis of a multi-element normalized diagram. The two sources require different amounts of sediment in the mantle wedge (LK 2\% versus HK 10\%) and a fluid component probably from altered ocean crust to explain the fluid mobile elements. Low-K Roccamonfina rocks are geochemically similar to those from Campi Flegrei, Ventotene, and Somma-Vesuvius, suggesting a similar proportion of sediment in their upper mantle source regions [3].

\section{Sampling and Analytical Techniques}

A representative suite of 300 volcanic rocks of samples was obtained on and around the flanks of Mount Somma-Vesuvius volcano, Roccamonfina volcano, Campi Flegrei Volcanic District and Ventotene Island on the basis of detailed field mapping (Rolandi, unpubl. Data); each rocks weighted $2 \mathrm{Kg}$, then was crushed to ash size, 2 $\mathrm{g}$ for each rocks were analyzed for $\mathrm{Pb}$ isotopic determinations. At least half of the samples are pumice and the rest are scoria and lava. Pb isotopes were determined at the US Geological Survey, Reston, VA, on a Finnigan-MAT 262 mass spectrometer. Pb isotopic analyses were done on 50 minerals separates (plagioclase, sanidine, and leucite). And 250 whole rocks (total of 300 samples, Table 1 and Table 2), using static multi-collection mode. Isotopes were separated using standard anion-exchange methods: $\mathrm{Pb}$ isotopic ratios were normalized for mass fractionation by $0.1 \%$ per mass unit on the basis of repeated runs of NBS-981; Pb blanks during 
Table 1. Pb isotopic composition of the volcanic rocks and minerals from Mt. Somma-Vesuvius volcano.

\begin{tabular}{|c|c|c|c|c|c|c|c|}
\hline Sample ID & & ${ }^{206} \mathrm{~Pb} /{ }^{204} \mathrm{~Pb}$ & SE(M)2 & ${ }^{207} \mathrm{~Pb} /{ }^{204} \mathrm{~Pb}$ & $\mathrm{SE}(\mathrm{M}) 2$ & ${ }^{208} \mathrm{~Pb} /{ }^{204} \mathrm{~Pb}$ & $\mathrm{SE}(\mathrm{M}) 2$ \\
\hline S1a & WR & 19.002 & 0.001 & 15.625 & 0.008 & 38.985 & 0.002 \\
\hline S1a & WR & 19.053 & 0.003 & 15.681 & 0.003 & 39.169 & 0.009 \\
\hline S1a & KSP & 19.127 & 0.042 & 15.752 & 0.036 & 39.349 & 0.092 \\
\hline S1b & WR & 19.013 & 0.003 & 15.633 & 0.003 & 39.014 & 0.007 \\
\hline S1b & WR & 19.041 & 0.002 & 15.664 & 0.001 & 39.121 & 0.005 \\
\hline S1b & KSP & 19.038 & 0.007 & 15.664 & 0.007 & 39.124 & 0.019 \\
\hline S1c & WR & 19.023 & 0.001 & 15.642 & 0.001 & 39.037 & 0.003 \\
\hline S1c & KSP & 19.039 & 0.002 & 15.661 & 0.002 & 39.105 & 0.006 \\
\hline S1d & WR & 19.002 & 0.0006 & 15.610 & 0.0005 & 38.935 & 0.001 \\
\hline S1d & WR & 18.994 & 0.002 & 15.607 & 0.001 & 38.925 & 0.003 \\
\hline S2(1) & WR & 19.030 & 0.005 & 15.621 & 0.005 & 39.032 & 0.035 \\
\hline S2(1) & WR & 19.033 & 0.003 & 15.629 & 0.003 & 38.986 & 0.007 \\
\hline S2(1) & KSP & 19.016 & 0.082 & 15.640 & 0.066 & 39.087 & 0.166 \\
\hline S2(2)a & WR & 19.042 & 0.006 & 15.637 & 0.005 & 39.013 & 0.014 \\
\hline $\mathrm{S} 2(2) \mathrm{a}$ & KSP & 19.538 & 0.678 & 16.040 & 0.552 & 39.915 & 1.459 \\
\hline $\mathrm{S} 2(2) \mathrm{b}$ & WR & 18.990 & 0.006 & 15.689 & 0.006 & 39.149 & 0.017 \\
\hline S2(2)b & WR & 18.931 & 0.002 & 15.611 & 0.001 & 38.893 & 0.003 \\
\hline S2(2)c & KSP & 18.982 & & 15.682 & & 39.135 & \\
\hline $\mathrm{S} 2(2) \mathrm{e}$ & WR & 18.985 & & 15.639 & & 38.987 & \\
\hline S2(3) & KSP & 18.979 & 0.003 & 15.666 & 0.003 & 39.085 & 0.007 \\
\hline S2(4) & WR & 18.958 & & 15.645 & & 39.024 & 0.003 \\
\hline S3(1)a & KSP & 18.949 & 0.001 & 15.626 & 0.001 & 38.950 & \\
\hline S3(1)b & WR & 18.966 & & 15.658 & & 39.067 & 0.004 \\
\hline S3(1)b & KSP & 18.950 & 0.001 & 15.629 & 0.001 & 38.955 & \\
\hline S3(1)c & WR & 18.949 & & 15.637 & & 38.995 & 0.004 \\
\hline S3(1)c & KSP & 18.954 & 0.001 & 15.635 & 0.001 & 38.978 & \\
\hline S3(1)d & WR & 19.004 & & 15.702 & & 39.223 & 0.009 \\
\hline S3(1)d & KSP & 18.947 & 0.004 & 15.627 & 0.003 & 38.952 & \\
\hline S3(1)e & WR & 18.935 & & 15.623 & & 38.946 & 0.004 \\
\hline S3(1)e & KSP & 18.968 & 0.002 & 15.652 & 0.001 & 39.043 & 0.006 \\
\hline S3(1)f & WR & 18.916 & 0.002 & 15.592 & 0.002 & 38.830 & \\
\hline S3(2)a & WR & 18.960 & & 15.647 & & 39.031 & 0.004 \\
\hline S3(2)b & WR & 18.941 & 0.002 & 15.615 & 0.001 & 38.914 & 0.002 \\
\hline S3(2)c & WR & 18.937 & 0.0007 & 15.613 & 0.0006 & 38.904 & 0.009 \\
\hline S3(2)c & WR & 18.909 & 0.005 & 15.597 & 0.003 & 38.851 & 0.004 \\
\hline S3(2)d & WR & 18.944 & 0.002 & 15.622 & 0.001 & 38.936 & 0.001 \\
\hline S3(2)e & WR & 18.936 & 0.0006 & 15.612 & 0.0005 & 38.901 & 0.007 \\
\hline S3(2)e & WR & 18.949 & 0.002 & 15.629 & 0.002 & 38.956 & \\
\hline
\end{tabular}


Continued

\begin{tabular}{|c|c|c|c|c|c|c|c|}
\hline S3(2)f & WR & 18.959 & & 15.645 & & 39.025 & 0.002 \\
\hline S3(3)a & WR & 18.940 & 0.001 & 15.614 & 0.001 & 38.908 & \\
\hline S3(3)a & WR & 18.975 & & 15.667 & & 39.098 & 0.004 \\
\hline S3(3)b & WR & 18.956 & 0.001 & 15.636 & 0.001 & 38.981 & \\
\hline S3(3)b & WR & 18.947 & & 15.646 & & 39.015 & \\
\hline S3(3)c & KSP & 18.956 & 0.001 & 15.638 & 0.001 & 38.984 & 0.004 \\
\hline S3(3)c & KSP & 18.975 & & 15.669 & & 39.101 & \\
\hline $\mathrm{S} 3(3) \mathrm{c}$ & WR & 18.931 & 0.0008 & 15.613 & 0.0008 & 38.902 & 0.002 \\
\hline S3(3)d & WR & 18.945 & 0.0006 & 15.624 & 0.0006 & 38.938 & 0.002 \\
\hline $\mathrm{S} 4(2) \mathrm{a}$ & WR & 18.936 & 0.0006 & 15.604 & 0.0006 & 38.874 & 0.001 \\
\hline $\mathrm{S} 4(2) \mathrm{a}$ & WR & 18.959 & 0.004 & 15.636 & 0.004 & 38.980 & 0.011 \\
\hline $\mathrm{S} 4(2) \mathrm{b}$ & WR & 18.963 & & 15.656 & & 39.051 & \\
\hline $\mathrm{S} 4(2) \mathrm{b}$ & WR & 18.936 & 0.0009 & 15.604 & 0.0008 & 38.874 & 0.002 \\
\hline $\mathrm{S} 4(4) \# 1$ & WR & 18.944 & 0.002 & 15.624 & 0.002 & 38.934 & 0.007 \\
\hline S5(1)b & WR & 19.053 & & 15.769 & & 39.430 & \\
\hline S5(1)c & WR & 19.034 & 0.006 & 15.737 & 0.006 & 39.312 & 0.018 \\
\hline S5(2)a & WR & 18.953 & 0.0007 & 15.629 & 0.0007 & 38.949 & 0.002 \\
\hline S5(2)a & WR & 18.960 & 0.004 & 15.647 & 0.004 & 39.015 & 0.014 \\
\hline $\mathrm{S} 5(2) \mathrm{a}$ & KSP & 19.001 & 0.005 & 15.684 & 0.005 & 39.140 & 0.016 \\
\hline $\mathrm{S} 5(2) \mathrm{a}$ & KSP & 19.020 & & 15.715 & & 39.257 & \\
\hline $\mathrm{S} 5(2) \mathrm{b}$ & WR & 18.967 & 0.001 & 15.648 & 0.001 & 39.011 & 0.004 \\
\hline $\mathrm{S} 5(2) \mathrm{b}$ & KSP & 18.979 & 0.002 & 15.657 & 0.002 & 39.050 & 0.007 \\
\hline S5(3)a & WR & 19.030 & & 15.736 & & 39.320 & \\
\hline $\mathrm{S} 5(3) \mathrm{b}$ & WR & 19.011 & 0.005 & 15.705 & 0.004 & 39.202 & 0.012 \\
\hline $\mathrm{S} 5(4) \mathrm{b}$ & WR & 18.953 & 0.002 & 15.609 & 0.002 & 38.887 & 0.005 \\
\hline S5(4)b & WR & 18.953 & 0.0009 & 15.615 & 0.0008 & 38.899 & 0.002 \\
\hline S6(1)a & WR & 18.953 & 0.0009 & 15.622 & 0.0008 & 38.927 & \\
\hline S6(1)a & KSP & 18.950 & 0.001 & 15.623 & 0.002 & 38.933 & 0.002 \\
\hline S6(1)b & WR & 18.933 & 0.001 & 15.603 & 0.001 & 38.863 & 0.006 \\
\hline S6(1)b & KSP & 18.944 & 0.002 & 15.618 & 0.002 & 38.912 & 0.003 \\
\hline S6(1)c & WR & 18.939 & 0.0008 & 15.611 & 0.0007 & 38.890 & 0.005 \\
\hline S6(1)c & KSP & 18.983 & 0.003 & 15.663 & 0.003 & 39.069 & 0.002 \\
\hline S6(2)a & WR & 18.971 & 0.002 & 15.662 & 0.002 & 39.051 & 0.008 \\
\hline S6(2)a & WR & 18.962 & 0.0008 & 15.640 & 0.0008 & 38.986 & 0.005 \\
\hline S6(2)a & KSP & 19.003 & 0.009 & 15.686 & 0.009 & 39.140 & 0.002 \\
\hline S9(1)a & KSP & 19.384 & 0.139 & 16.041 & 0.114 & 40.168 & 0.027 \\
\hline S9(1)a & KSP & 18.995 & & 15.688 & & 39.148 & 0.298 \\
\hline S9(1)b & KSP & 18.978 & & 15.664 & & 39.084 & \\
\hline s9(1)b & Sink & 18.959 & 0.001 & 15.633 & 0.001 & 38.967 & \\
\hline
\end{tabular}


A. Paone

\section{Continued}

\begin{tabular}{|c|c|c|c|c|c|c|c|}
\hline S9(1)c & KSP & 18.987 & & 15.683 & & 39.146 & 0.003 \\
\hline S9(1)d & WR & 18.947 & 0.001 & 15.618 & 0.0009 & 38.916 & \\
\hline S10(1)a & KSP & 18.963 & 0.002 & 15.634 & 0.002 & 38.960 & 0.002 \\
\hline S10(1)a & KSP & 18.982 & & 15.666 & & 39.076 & 0.005 \\
\hline $\mathrm{S} 10(1) \mathrm{C}$ & KSP & 19.010 & & 15.687 & & 39.161 & \\
\hline S10(1)d & WR & 18.959 & 0.001 & 15.608 & 0.001 & 38.887 & \\
\hline S11(1) & WR & 18.948 & 0.0007 & 15.622 & 0.0006 & 38.913 & 0.003 \\
\hline $\mathrm{S} 12(2)$ & WR & 18.982 & & 15.695 & & 39.174 & 0.0016 \\
\hline S13(1)a & WR & 18.963 & 0.008 & 15.664 & 0.007 & 39.057 & \\
\hline S13(1)a & WR & 18.963 & & 15.664 & & 39.057 & 0.017 \\
\hline $\mathrm{S} 13(1) \mathrm{b}$ & WR & 18.940 & 0.001 & 15.609 & 0.001 & 38.881 & \\
\hline $\mathrm{S} 13(2) \mathrm{b}$ & WR & 18.974 & & 15.673 & & 39.099 & 0.003 \\
\hline $\mathrm{S} 14(1)$ & WR & 18.955 & 0.002 & 15.641 & 0.002 & 38.982 & \\
\hline $\mathrm{S} 14(2)$ & WR & 18.968 & & 15.654 & & 39.032 & 0.006 \\
\hline S15(1)b & WR & 18.949 & 0.003 & 15.623 & 0.003 & 38.915 & \\
\hline $\mathrm{S} 15(1) \mathrm{b}$ & WR & 19.017 & & 15.720 & & 39.261 & 0.008 \\
\hline S15(2)a & WR & 18.998 & 0.022 & 15.689 & 0.018 & 39.143 & \\
\hline S16(1) & WR & 19.044 & & 15.753 & & 39.370 & 0.044 \\
\hline S17(1)a & WR & 19.025 & 0.006 & 15.722 & 0.006 & 39.252 & \\
\hline $\mathrm{S} 17(2) \mathrm{b}$ & WR & 18.929 & 0.001 & 15.606 & 0.0008 & 38.8613 & 0.021 \\
\hline S18(1)a & WR & 18.901 & 0.002 & 15.585 & 0.001 & 38.796 & 0.002 \\
\hline S18(1)b & WR & 18.949 & 0.0008 & 15.622 & 0.0008 & 38.9139 & 0.005 \\
\hline S19(1)a & WR & 19.088 & 0.071 & 15.780 & 0.059 & 39.400 & 0.002 \\
\hline $\mathrm{S} 20$ & WR & 19.070 & 0.004 & 15.655 & 0.003 & 39.068 & 0.147 \\
\hline S20 & WR & 19.089 & & 15.687 & & 39.185 & 0.01 \\
\hline S21(1)a & WR & 19.024 & 0.024 & 15.646 & 0.02 & 39.022 & \\
\hline S21(1)a & WR & 19.043 & & 15.677 & & 39.139 & 0.05 \\
\hline S21(1)b & WR & 19.030 & 0.012 & 15.677 & 0.011 & 39.137 & \\
\hline S21(1)b & WR & 19.049 & & 15.709 & & 39.254 & 0.026 \\
\hline S21(1)c & WR & 18.972 & 0.001 & 15.614 & 0.0009 & 38.906 & \\
\hline S21(1)c & WR & 19.035 & 0.008 & 15.668 & 0.007 & 39.094 & 0.002 \\
\hline S21(1)c & WR & 19.054 & & 15.700 & & 39.212 & 0.019 \\
\hline S21(2)C & WR & 19.125 & 0.138 & 15.763 & 0.109 & 39.327 & \\
\hline S21(2)d & WR & 19.038 & 0.006 & 15.673 & 0.005 & 39.113 & 0.276 \\
\hline S21(2)d & WR & 19.058 & & 15.704 & & 39.231 & 0.0145 \\
\hline S21(2)e & WR & 18.980 & 0.0009 & 15.602 & 0.0008 & 38.869 & \\
\hline S21(2)f & WR & 18.997 & 0.001 & 15.615 & 0.001 & 38.916 & 0.002 \\
\hline S21(2)f & WR & 19.016 & & 15.646 & & 39.032 & 0.004 \\
\hline V1a & WR & 18.987 & 0.002 & 15.614 & 0.002 & 38.911 & \\
\hline
\end{tabular}




\section{Continued}

\begin{tabular}{|c|c|c|c|c|c|c|c|}
\hline R 1a & WR & 19.006 & & 15.646 & & 39.028 & 0.005 \\
\hline V1b & WR & 19.020 & 0.05 & 15.647 & 0.041 & 39.021 & \\
\hline $\mathrm{R} 1 \mathrm{~b}$ & WR & 19.040 & & 15.679 & & 39.138 & 0.102 \\
\hline V1c & WR & 19.032 & 0.003 & 15.677 & 0.004 & 39.111 & \\
\hline R 1c & WR & 19.051 & & 15.708 & & 39.229 & 0.012 \\
\hline V2a & WR & 19.096 & 0.09 & 15.740 & 0.079 & 39.272 & \\
\hline $\mathrm{R} 2 \mathrm{~b}$ & WR & 18.985 & & 15.646 & & 39.015 & 0.15 \\
\hline V2b & WR & 18.966 & 0.001 & 15.615 & 0.001 & 38.898 & \\
\hline V3a & WR & 18.965 & 0.002 & 15.593 & 0.002 & 38.852 & 0.003 \\
\hline R 3a & WR & 18.984 & & 15.625 & & 38.969 & 0.005 \\
\hline V3b & WR & 19.054 & 0.004 & 15.656 & 0.002 & 39.076 & \\
\hline $\mathrm{R} 3 \mathrm{~b}$ & WR & 19.073 & & 15.688 & & 39.193 & 0.01 \\
\hline V4 & WR & 18.989 & 0.002 & 15.652 & 0.002 & 39.015 & \\
\hline R 4 & WR & 19.008 & & 15.683 & & 39.132 & 0.006 \\
\hline V5 & WR & 19.234 & 0.2 & 15.897 & 0.164 & 39.622 & \\
\hline V6a & WR & 18.935 & 0.001 & 15.586 & 0.001 & 38.799 & 0.42 \\
\hline R 6a & WR & 18.954 & & 15.617 & & 38.915 & 0.003 \\
\hline V6b & WR & 18.977 & 0.013 & 15.639 & 0.011 & 38.982 & \\
\hline R 6b & WR & 18.996 & & 15.670 & & 39.099 & 0.028 \\
\hline V6c & WR & 18.979 & 0.009 & 15.638 & 0.008 & 38.976 & \\
\hline R 6c & WR & 18.998 & & 15.669 & & 39.093 & 0.023 \\
\hline V7 & WR & 19.190 & 0.199 & 15.812 & 0.163 & 39.402 & \\
\hline V7 & WR & 19.024 & 0.001 & 15.630 & 0.001 & 38.885 & 0.413 \\
\hline V8 & WR & 18.929 & 0.008 & 15.612 & 0.007 & 38.874 & 0.003 \\
\hline R 8 & WR & 18.948 & & 15.643 & & 38.991 & 0.017 \\
\hline V9a & WR & 18.985 & 0.01 & 15.629 & 0.009 & 38.932 & \\
\hline R 9a & WR & 19.004 & & 15.661 & & 39.049 & 0.022 \\
\hline V9b & WR & 18.967 & 0.003 & 15.614 & 0.002 & 38.898 & \\
\hline R 9b & WR & 18.986 & & 15.645 & & 39.015 & 0.008 \\
\hline V10 & WR & 19.101 & 0.076 & 15.677 & 0.062 & 39.134 & \\
\hline R 10 & WR & 19.120 & & 15.709 & & 39.252 & 0.152 \\
\hline V14 & WR & 19.005 & 0.008 & 15.602 & 0.007 & 38.894 & \\
\hline V14 & WR & 19.024 & & 15.633 & & 39.011 & 0.017 \\
\hline V18 & WR & 18.999 & 0.009 & 15.595 & 0.008 & 38.867 & \\
\hline V18 & WR & 19.018 & & 15.627 & & 38.984 & 0.02 \\
\hline V21 & WR & 18.968 & 0.004 & 15.633 & 0.003 & 38.953 & \\
\hline V21 & WR & 18.987 & & 15.664 & & 39.070 & 0.008 \\
\hline V23 & WR & 18.968 & 0.006 & 15.636 & 0.005 & 38.976 & \\
\hline V23 & WR & 18.970 & 0.001 & 15.635 & 0.0009 & 38.954 & 0.014 \\
\hline
\end{tabular}




\section{Continued}

\begin{tabular}{|c|c|c|c|c|c|c|c|}
\hline V23 & WR & 18.987 & & 15.667 & & 39.093 & 0.002 \\
\hline V30 & WR & 18.954 & 0.001 & 15.616 & 0.0008 & 38.900 & \\
\hline V34 & WR & 18.980 & 0.003 & 15.659 & 0.002 & 39.054 & 0.002 \\
\hline V35 & WR & 18.985 & 0.004 & 15.651 & 0.004 & 39.031 & 0.015 \\
\hline V35 & WR & 19.004 & & 15.683 & & 39.148 & \\
\hline V42 & WR & 18.952 & 0.002 & 15.621 & 0.001 & 38.915 & 0.004 \\
\hline V42 & WR & 19.013 & 0.018 & 15.683 & 0.016 & 39.119 & 0.049 \\
\hline V42 & WR & 19.032 & & 15.714 & & 39.236 & \\
\hline V46 & WR & 18.963 & 0.001 & 15.630 & 0.001 & 38.935 & 0.003 \\
\hline V46 & WR & 18.982 & & 15.661 & & 39.052 & \\
\hline V59 & WR & 19.030 & & 15.724 & & 39.279 & \\
\hline V64 & WR & 18.991 & 0.002 & 15.632 & 0.002 & 38.948 & 0.005 \\
\hline V84 & WR & 18.959 & 0.001 & 15.614 & 0.001 & 38.901 & 0.002 \\
\hline V84 & WR & 18.946 & 0.0008 & 15.604 & 0.0008 & 38.856 & 0.002 \\
\hline V84 & WR & 18.942 & 0.003 & 15.601 & 0.003 & 38.844 & 0.008 \\
\hline V94 & WR & 19.178 & & 15.767 & & 39.435 & \\
\hline V97 & WR & 19.036 & 0.002 & 15.615 & 0.002 & 38.950 & 0.05 \\
\hline V97 & WR & 19.036 & 0.003 & 15.616 & 0.002 & 38.951 & 0.006 \\
\hline V102 & WR & 18.979 & 0.001 & 15.628 & 0.001 & 38.936 & 0.003 \\
\hline V102 & WR & 19.050 & & 15.717 & & 39.228 & \\
\hline V102 & WR & 19.031 & 0.029 & 15.685 & 0.026 & 39.110 & 0.067 \\
\hline V110 & WR & 18.964 & 0.001 & 15.602 & 0.0009 & 38.851 & 0.003 \\
\hline V141 & WR & 19.056 & 0.001 & 15.640 & 0.001 & 39.032 & 0.003 \\
\hline V155 & WR & 18.964 & 0.001 & 15.602 & 0.0009 & 38.851 & 0.003 \\
\hline SCL1 & WR & 18.988 & 0.002 & 15.602 & 0.002 & 38.902 & 0.007 \\
\hline SCD1 & WR & 18.981 & 0.003 & 15.607 & 0.003 & 38.891 & 0.009 \\
\hline SCL1a & WR & 19.008 & 0.003 & 15.618 & 0.003 & 38.955 & 0.012 \\
\hline SCL3 & WR & 19.023 & 0.005 & 15.628 & 0.005 & 39.019 & 0.015 \\
\hline SCL4 & WR & 18.993 & 0.002 & 15.621 & 0.002 & 38.965 & 0.008 \\
\hline SCL5 & WR & 18.989 & 0.002 & 15.617 & 0.002 & 38.959 & 0.004 \\
\hline SCL6 & WR & 18.989 & 0.002 & 15.642 & 0.001 & 39.033 & 0.004 \\
\hline SCD2\#1 & WR & 19.077 & 0.003 & 15.604 & 0.002 & 38.975 & 0.007 \\
\hline $\mathrm{S} 2(2) \mathrm{C}$ & KSP & 18.963 & 0.002 & 15.650 & 0.003 & 39.018 & 0.008 \\
\hline S3(3)6 & KSP & 18.943 & 0.003 & 15.632 & 0.002 & 38.965 & 0.006 \\
\hline
\end{tabular}

this study were $-1 \mathrm{ng}$. The $\mathrm{Pb}$ isotopic data have a total uncertainty of $0.1 \%$ (2 s).

\section{Discussion}

The $\mathrm{Pb}$ isotopic composition is presented from mainly the Somma-Vesuvius volcano and cover the eruption ages from Somma caldera activity [ $<39 \mathrm{Ka}$ (age of the Campanian Ignimbrite)] until the last vulcanian effusive eruption of 1944 AD (Table 3, [12], references therein). Then, a representative sample collection of the Campi Fle- 
Table 2. Pb isotopic composition of a representative suite of volcanic rocks from Campi Flegrei District (CF), Roccamonfina (RM), Campi Flegrei Breccia Museo (CFb), Ignimbrite Campana (ICB, sanidine and whole rocks: ALT, MTF, SFC, Ve, Sa, Mo, MP), Somma caldera (SCL, SCD), Ventotene xenoliths (Vt).

\begin{tabular}{|c|c|c|c|c|c|c|c|c|c|c|}
\hline Samples ID & & Petrography & AGE & Lithotype & $\begin{array}{l}\text { Pb206/ } \\
\text { Pb204 }\end{array}$ & 2SE(M) & $\begin{array}{l}\text { Pb207/ } \\
\text { Pb204 }\end{array}$ & 2SE(M) & $\begin{array}{l}\mathrm{Pb} 208 / \\
\mathrm{Pb} 204\end{array}$ & 2SE(M) \\
\hline RM 1 & Whole rock & Leuc-theph & $1.5-0.3 \mathrm{MA}$ & Lava & 18.866 & 0.011 & 15.749 & 0.009 & 39.268 & 0.026 \\
\hline RM 1 & Separate & Ksp & & & 18.739 & 0.001 & 15.612 & 0.001 & 38.762 & 0.003 \\
\hline RM 3 & Whole rock & K-Basalt & $0.3 \mathrm{MA}$ & Lava & 18.943 & 0.006 & 15.617 & 0.005 & 38.968 & 0.016 \\
\hline RM 3' & Whole rock & & & & 19.167 & 0.004 & 15.683 & 0.003 & 39.209 & 0.01 \\
\hline RM 5 & Whole rock & K-Basalt & $0.3 \mathrm{MA}$ & Lava & 18.827 & 0.003 & 15.684 & 0.003 & 39.048 & 0.009 \\
\hline RM 6 & Whole rock & K-Basalt & $0.3 \mathrm{MA}$ & Lava & 18.839 & 0.003 & 15.680 & 0.003 & 39.039 & 0.009 \\
\hline RM 7 & Whole rock & Leuc-tephr & $1.5-0.3 \mathrm{MA}$ & Lava & 18.716 & 0.004 & 15.605 & 0.003 & 38.784 & 0.008 \\
\hline RM 7 & Separate & Ksp & & & 18.749 & 0.002 & 15.608 & 0.001 & 38.794 & 0.003 \\
\hline RM 8 & Whole rock & K-basalt & 0.3 MA? & Lava & 19.119 & 0.002 & 15.677 & 0.002 & 39.177 & 0.005 \\
\hline RM 10 & Whole rock & Latite & $0.3 \mathrm{MA}$ & $\begin{array}{c}\text { Spatter } \\
\text { cone? }\end{array}$ & 19.093 & 0.002 & 15.678 & 0.002 & 39.173 & 0.008 \\
\hline RM 12 & Whole rock & Trachibasalt & $0.3 \mathrm{MA} ?$ & lava & 18.884 & 0.003 & 15.687 & 0.007 & 39.080 & 0.018 \\
\hline CF X & Whole rock & K-Basalt & & lava & 19.201 & 0.0009 & 15.630 & 0.0007 & 39.116 & 0.002 \\
\hline CF 1 & Whole rock & Trachibasalt & >40 ky BP & Scoria & 19.032 & 0.004 & 15.620 & 0.002 & 38.948 & 0.008 \\
\hline CF 2 & Whole rock & Trachibasalt & 40 - 17 ky BP & Scoria & 19.126 & 0.0006 & 15.615 & 0.0005 & 39.002 & 0.006 \\
\hline CF 3 & Whole rock & Trachibasalt & 17,000 у BP & Scoria & 18.970 & 0.001 & 15.593 & 0.001 & 38.837 & 0.003 \\
\hline CF 5 & Whole rock & Latite & $?$ & Scoria & 18.971 & 0.002 & 15.628 & 0.001 & 38.950 & 0.004 \\
\hline CF 6 & Whole rock & Latite & 14,000 y BP & Scoria & 19.012 & 0.003 & 15.647 & 0.003 & 39.042 & 0.009 \\
\hline CF 7 & Whole rock & Trachyte & 10,000 у ВР & Pomice & 18.963 & 0.0007 & 15.666 & 0.0006 & 38.909 & 0.002 \\
\hline CF 8 & Whole rock & Latite & $40-10$ ky BP & Pomice & 18.989 & 0.002 & 15.638 & 0.002 & 38.990 & 0.007 \\
\hline CF 9 & Whole rock & Latite & 10 - 4 ky BP & Pomice & 18.922 & 0.002 & 15.650 & 0.003 & 38.965 & 0.006 \\
\hline CF 10 & Whole rock & Trachibasalt & 8 - 5 ky BP & Pomice & 18.936 & 0.001 & 15.580 & 0.0009 & 38.798 & 0.002 \\
\hline \multicolumn{11}{|l|}{ CF 10} \\
\hline CFb 1 & Whole rock & Basalt & & Lava & 18.974 & 0.002 & 15.684 & 0.002 & 39.082 & 0.008 \\
\hline CFb 2 & Whole rock & Basalt & & Lava & 18.953 & 0.002 & 15.609 & 0.001 & 38.855 & 0.004 \\
\hline CFb 1 & Ksp & Basalt & & & 19.044 & 0.002 & 15.626 & 0.002 & 38.934 & 0.005 \\
\hline CFb 2 & Ksp & Basalt & & & 19.133 & 0.002 & 15.615 & 0.001 & 39.026 & 0.004 \\
\hline CFb 3 & Ksp & Leuc-tephr. & & & 19.277 & 0.001 & 15.659 & 0.0009 & 39.211 & 0.003 \\
\hline CFb 4 & Ksp & Leuc-tephr. & & & 19.241 & 0.002 & 15.641 & 0.001 & 39.138 & 0.004 \\
\hline ICB 1 & Separate & Sanidine & 37 - 33 ky BP & & 19.085 & 0.002 & 15.616 & 0.002 & 39.011 & 0.004 \\
\hline ICB 2 & Separate & Sanidine & 37 - 33 ky BP & & 19.159 & 0.003 & 15.695 & 0.004 & 39.266 & 0.014 \\
\hline ICB 3 & Separate & Sanidine & 37 - 33 ky BP & & 19.136 & 0.005 & 15.663 & 0.003 & 39.179 & 0.008 \\
\hline ICB 4 & Separate & Sanidine & 37 - 33 ky BP & & 19.136 & 0.005 & 15.663 & 0.003 & 39.061 & 0.003 \\
\hline ICB 5 & Separate & Sanidine & 37 - 33 ky BP & & 19.102 & 0.001 & 15.635 & 0.001 & 39.064 & 0.004 \\
\hline ICB HB6a & Separate & Sanidine & 37 - 33 ky BP & & 19.112 & 0.001 & 15.649 & 0.001 & 39.118 & 0.005 \\
\hline ICB HB6b & Separate & Sanidine & 37 - 33 ky BP & & 19.114 & 0.0007 & 15.642 & 0.0005 & 39.085 & 0.001 \\
\hline ICB HB7a & Separate & Sanidine & 37 - 33 ky BP & & 19.122 & 0.0007 & 15.627 & 0.0006 & 39.049 & 0.001 \\
\hline ICB 8 & Separate & Sanidine & 37 - 33 ky BP & & 19.023 & 0.003 & 15.629 & 0.002 & 39.044 & 0.006 \\
\hline ICB 9 & Separate & Sanidine & 37 - 33 ky BP & & 19.128 & 0.0009 & 15.635 & 0.0007 & 39.078 & 0.002 \\
\hline ICB 9sec & Separate & Sanidine & 37 - 33 ky BP & & 19.150 & 0.007 & 15.678 & 0.006 & 39.226 & 0.006 \\
\hline ICB 10 & Separate & Sanidine & 37 - 33 ky BP & & 19.133 & 0.0007 & 15.650 & 0.0006 & 39.122 & 0.001 \\
\hline ICB 11 & Separate & Sanidine & 37 - 33 ky BP & & 19.093 & 0.0009 & 15.625 & 0.0009 & 39.026 & 0.003 \\
\hline ICB 12 & Separate & Sanidine & 37 - 33 ky BP & & 19.108 & 0.0009 & 15.623 & 0.0008 & 39.046 & 0.002 \\
\hline ICB 13 & Separate & Sanidine & 37 - 33 ky BP & & 19.106 & 0.001 & 15.625 & 0.001 & 39.037 & 0.003 \\
\hline
\end{tabular}




\section{Continued}

\begin{tabular}{|c|c|c|c|c|c|c|c|c|c|c|}
\hline ICB 13sec & Separate & Sanidine & 37 - 33 ky BP & & 19.118 & 0.0009 & 15.637 & 0.0008 & 39.076 & 0.002 \\
\hline ICB 14 & Separate & Sanidine & 37 - 33 ky BP & & 19.097 & 0.0008 & 15.621 & 0.0006 & 39.020 & 0.002 \\
\hline ICB 15 & Separate & Sanidine & 37 - 33 ky BP & & 19.095 & 0.006 & 15.620 & 0.005 & 39.024 & 0.012 \\
\hline ALT 1 & Separate & Sanidine & 37 - 33 ky BP & & 19.108 & 0.001 & 15.631 & 0.0008 & 39.056 & 0.002 \\
\hline ALT 2 & Separate & Sanidine & 37 - 33 ky BP & & 19.147 & 0.0007 & 15.670 & 0.0006 & 39.190 & 0.002 \\
\hline ALT 3 & Separate & Sanidine & 37 - 33 ky BP & & 19.115 & 0.0009 & 15.642 & 0.0009 & 39.090 & 0.003 \\
\hline ALT 4 & Separate & Sanidine & 37 - 33 ky BP & & 19.103 & 0.0006 & 15.629 & 0.0004 & 39.047 & 0.001 \\
\hline Ponti Rossi & Separate & Sanidine & 37 - 33 ky BP & & 19.095 & 0.001 & 15.634 & 0.0007 & 39.055 & 0.002 \\
\hline MTF 1 & Separate & Sanidine & 37 - 33 ky BP & & 19.118 & 0.0006 & 15.642 & 0.0005 & 39.090 & 0.001 \\
\hline $\begin{array}{l}\text { Pian } \\
\text { Sorren. }\end{array}$ & Separate & Sanidine & 37 - 33 ky BP & & 19.128 & 0.0007 & 15.633 & 0.0006 & 39.070 & 0.002 \\
\hline SFC 1a & Separate & Sanidine & 37 - 33 ky BP & & 19.094 & 0.0006 & 15.631 & 0.0006 & 39.045 & 0.002 \\
\hline SFC 2 & Separate & Sanidine & 37 - 33 ky BP & & 19.109 & 0.0008 & 15.630 & 0.0008 & 39.051 & 0.002 \\
\hline Ve 1 & Separate & Sanidine & 37 - 33 ky BP & & 19.121 & 0.0005 & 15.628 & 0.0004 & 39.054 & 0.0009 \\
\hline Sa 1b & Separate & Sanidine & 37 - 33 ky BР & & 19.112 & 0.0007 & 15.625 & 0.0006 & 39.038 & 0.001 \\
\hline Mo 1 & Separate & Sanidine & 37 - 33 ky BP & & 19.083 & 0.001 & 15.602 & 0.001 & 38.961 & 0.004 \\
\hline Mo 2 & Separate & Sanidine & 37 - 33 ky BP & & 19.106 & 0.0009 & 15.632 & 0.0007 & 39.053 & 0.002 \\
\hline MP 1 & Separate & Sanidine & 37 - 33 ky BP & & 19.144 & 0.001 & 15.657 & 0.001 & 39.151 & 0.003 \\
\hline MP 1 & Separate & Sanidine & 37 - 33 ky BP & & 19.154 & 0.0008 & 15.668 & 0.0007 & 39.185 & 0.002 \\
\hline MP 2a & Separate & Sanidine & 37 - 33 ky BP & & 19.120 & 0.0008 & 15.626 & 0.0006 & 39.049 & 0.002 \\
\hline MP 2b & Separate & Sanidine & 37 - 33 ky BP & & 19.109 & 0.001 & 15.635 & 0.0008 & 39.067 & 0.002 \\
\hline MPI-WB & whole rock & & 37 - 33 ky BP & & 19.137 & 0.001 & 15.651 & 0.001 & 39.133 & 0.003 \\
\hline VE-1-WB & Whole rock & & 37 - 33 ky BP & & 19.166 & 0.003 & 15.671 & 0.003 & 39.194 & 0.008 \\
\hline ALT-1-WB & Whole rock & & 37 - 33 ky BP & & 19.113 & 0.002 & 15.639 & 0.002 & 39.080 & 0.007 \\
\hline Sa 1a-WB & Whole rock & & 37 - 33 ky BP & & 19.072 & 0.003 & 15.617 & 0.002 & 39.067 & 0.007 \\
\hline Sa 1b-WB & Whole rock & & 37 - 33 ky BP & & 19.132 & 0.0004 & 15.652 & 0.0003 & 39.129 & 0.0009 \\
\hline ICB 9-WB & Whole rock & & 37 - 33 ky BP & & 19.148 & 0.001 & 15.656 & 0.001 & 39.151 & 0.005 \\
\hline SCL 1 & Whole rock & & 35 - 25 ky BP & Lava & 18.988 & 0.002 & 15.602 & 0.002 & 38.902 & 0.007 \\
\hline SCL 1a & Whole rock & & 35 - 25 ky BP & & 19.008 & 0.003 & 15.618 & 0.003 & 38.955 & 0.012 \\
\hline SCL 3 & Whole rock & & 35 - 25 ky BP & & 19.023 & 0.005 & 15.628 & 0.005 & 39.019 & 0.015 \\
\hline SCL 4 & Whole rock & & 35 - 25 ky BP & Lava & 18.993 & 0.002 & 15.621 & 0.002 & 38.965 & 0.008 \\
\hline SCL 5 & Whole rock & & 35 - 25 ky BP & & 18.989 & 0.002 & 15.617 & 0.002 & 38.959 & 0.004 \\
\hline SCL 6 & Whole rock & & 35 - 25 ky BP & & 18.989 & 0.002 & 15.642 & 0.001 & 39.033 & 0.004 \\
\hline SCD \#2-1 & Whole rock & & S.Cal. Dykes & & 19.077 & 0.003 & 15.604 & 0.002 & 38.975 & 0.003 \\
\hline Vt 1 & Whole rock & Xenlith. & & & 18.815 & 0.005 & 15.622 & 0.005 & 38.802 & 0.012 \\
\hline Vt 2 & Whole rock & Xenlith. & & & 18.538 & 0.007 & 15.603 & 0.006 & 38.506 & 0.019 \\
\hline Vt 3b & whole rock & Xenlith. & & & 18.835 & 0.003 & 15.633 & 0.003 & 38.810 & 0.007 \\
\hline Vt 4 & Whole rock & Xenolith. & & & 18.757 & 0.001 & 15.637 & 0.001 & 38.857 & 0.003 \\
\hline Vt 4 & Ksp & Xenolith. & & & 18.765 & 0.001 & 15.647 & 0.001 & 38.888 & 0.003 \\
\hline Vt 5a & Whole rock & Xenlith. & & & 18.742 & 0.002 & 15.623 & 0.001 & 38.810 & 0.003 \\
\hline Vt 5a & Ksp & Xenolith. & & & 18.753 & 0.0008 & 15.629 & 0.0007 & 38.832 & 0.002 \\
\hline Vt 5b & Ksp & Xenolith. & & & 18.737 & 0.002 & 15.621 & 0.002 & 38.804 & 0.004 \\
\hline Vt 6 & Whole rock & Xenolith. & & & 18.742 & 0.002 & 15.653 & 0.002 & 38.873 & 0.005 \\
\hline
\end{tabular}

grei Volcanic District (Campi Flegrei, Procida and Ischia) is also presented. A detailed sampling especially for the separate sanidine very much present in the products of this eruption and several representative whole rocks (age of Campanian Ignimbrite eruption: $39 \mathrm{Ka}$, [13], Figure 2) of the Campanian Ignimbrite eruption and several representative analyses of Roccamonfina volcano [3] and Ventotene xenoliths [14] are also presented. The ${ }^{207} \mathrm{~Pb} /{ }^{204} \mathrm{~Pb}$ versus ${ }^{206} \mathrm{~Pb} /{ }^{204} \mathrm{~Pb}$ diagram is presented with all the above data and the following end members 
[MORB [15] [16], Hercynian basement-Calabrian crust [17] [18], all the data worldwide of the shallow sediments and GLOSS (Global Sediment Subducting [19], Plank and Langmuir, 1998), Archean upper crust [16] (Figure 3)]. From the Pb-Pb diagram, I envisage that the all Campanian Volcanic Province is formed by three

Table 3. Eruption, age, and explosive tipology of Plinian and interplinian events in the last $25 \mathrm{ky}$.

\begin{tabular}{|c|c|c|c|c|c|}
\hline Formation & Age & Type of activity & Magmatic groups & $\begin{array}{l}\text { Dispersion } \\
\text { direction }\end{array}$ & $\begin{array}{l}\text { Volume } \\
\left(\mathrm{km}^{3}\right)\end{array}$ \\
\hline Codola & 25 ka BP & Plinian & I group & S-E & 1.4 \\
\hline Post-Codola & 25 - 17 ka BP & Strombolian and effusive & I group & & \\
\hline Sarno & $17 \mathrm{ka} \mathrm{BP}$ & Plinian & I group & E & $4.4-6$ \\
\hline Post-Sarno & 17 - 16 ka BP & Strombolian and effusive & I group & & \\
\hline Novelle-Seggiari-Bosco & 15 - 14 ka BP & Plinian & I group & $\mathrm{N}-\mathrm{NE}$ & 1.5 \\
\hline Ottaviano & 8 ka BP & Plinian & II group & E-NE & 2.4 \\
\hline Avellino & $3800 \mathrm{BP}$ & Plinian & II group & E-NE & 2.5 \\
\hline Protohistorical & 3800 - 2700 BP & Vulcanian and strombolian & II group & & \\
\hline Pompei AD 79 & $79 \mathrm{AD}$ & Plinian & III group & E-SE & $\sim 4$ \\
\hline Ancient historical & $79-203 \mathrm{AD}$ & $\begin{array}{c}\text { Vulcanian, strombolian and } \\
\text { effusive }\end{array}$ & III group & & \\
\hline Pollena AD 472 & $472 \mathrm{AD}$ & Plinian & III group & N-NE & 1.2 \\
\hline Medieval & $472-1139 \mathrm{AD}$ & $\begin{array}{l}\text { Vulcanian, strombolian and } \\
\text { effusive }\end{array}$ & III group & & \\
\hline 1631 & $1631 \mathrm{AD}$ & Plinian & III group & $\mathrm{N}-\mathrm{NE}$ & 1.1 \\
\hline $1631-1944$ & $1631-1944 \mathrm{AD}$ & $\begin{array}{l}\text { Vulcanian, strombolian and } \\
\text { effusive }\end{array}$ & III group & & \\
\hline
\end{tabular}

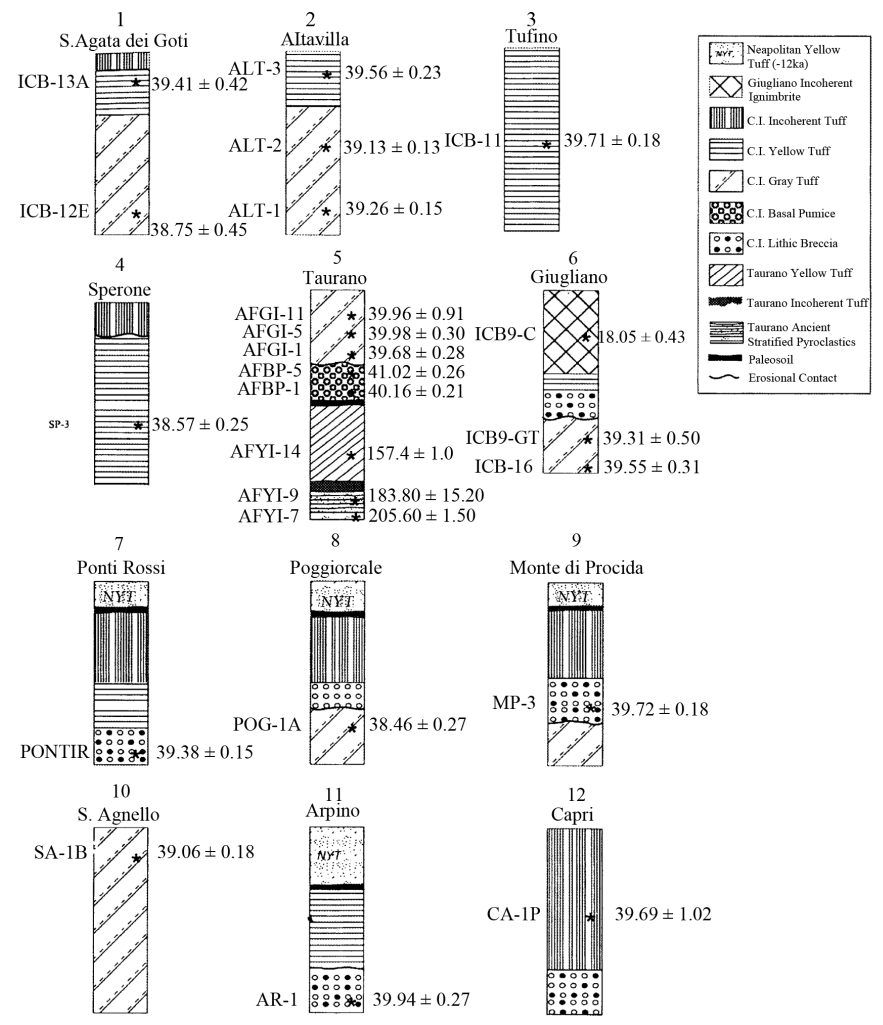

Figure 2. Schematic stratigraphic columns of the Campanian Ignimbrites. Ages are ka and are based on incremental-heating ${ }^{40} \mathrm{Ar}{ }^{39} \mathrm{Ar}$ analyses of pure sanidine mineral separates (modified from [13]). 


\section{Campanian Volcanic Province}

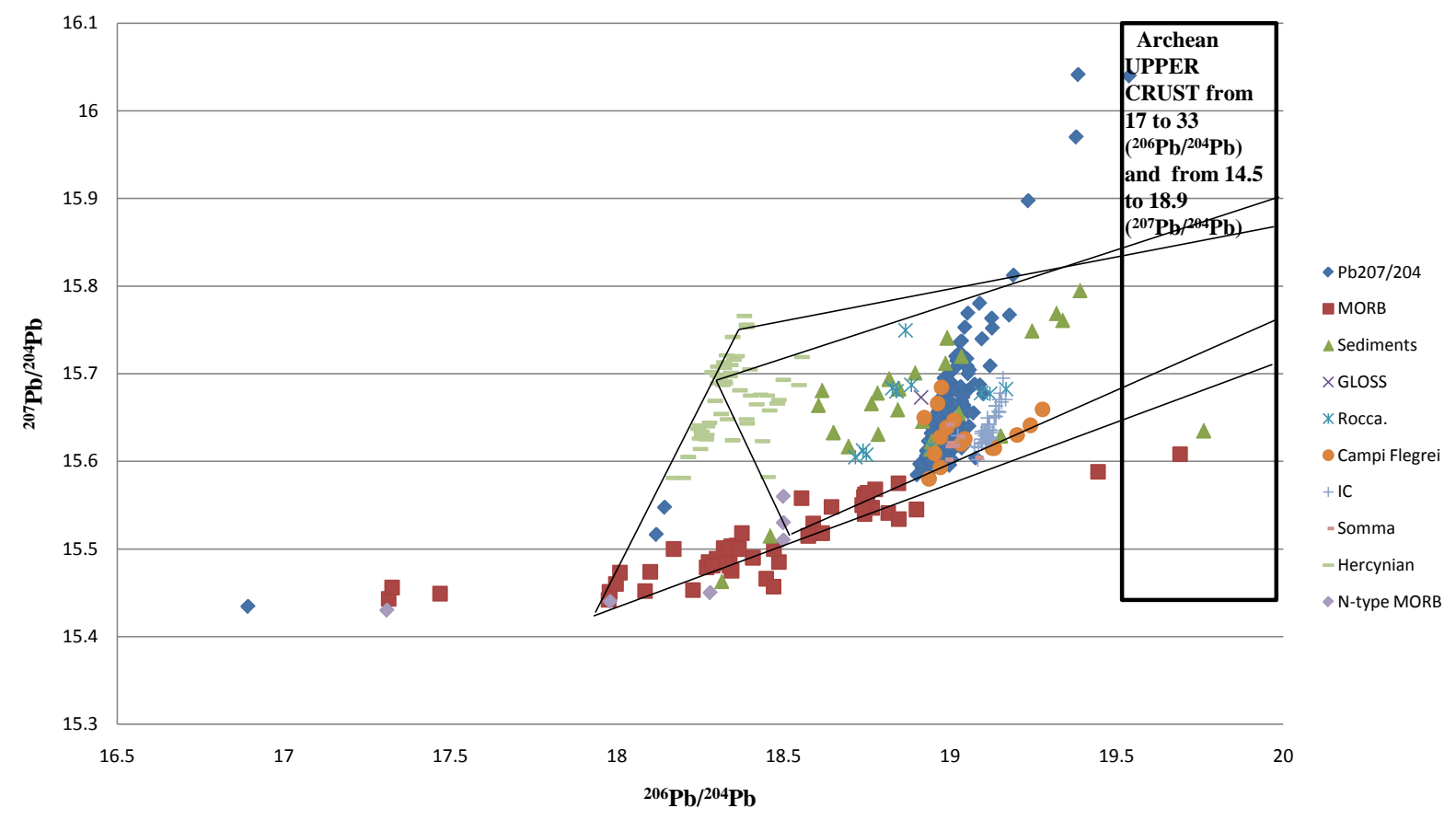

Figure 3. The ${ }^{207} \mathrm{~Pb} /{ }^{204} \mathrm{~Pb}$ versus ${ }^{206} \mathrm{~Pb} /{ }^{204} \mathrm{~Pb}$ diagram is presented with all the above data and the following end members [MORB [15] [16], Hercynian basement-Calabrian crust [17] [18], all the data worldwide of the shallow sediments and GLOSS (Global Sediment Subducting [19], Archean upper crust [16])].

end-menbers: 1) MORB, 2) Hercynian basement, 3) upper crust. This can be computed as I show with different styles of mixing duo to the large range of the end members. Anyhow, the result and interpretation does not change much. The model that I develop is that these volcanic products are produced by signatures of old arching upper crust and then assimilated with the Hercynian basement. Finally, I can also state that the recent sediments [19] are an average between the Hercynian component and the Archean upper crust as can be suggested by the past plate tectonic and the rock cycles.

\section{Conclusion}

Finally, I can say that the Campanian volcanic products are produced by a MORB component that has been contaminated by a series of upper crust component [20]. Anyhow, I can assume that the distinctive isotopic trend has also been influenced by an Archean upper crust. The magma has also undergone to storage and melting of a Hercynian component as testified by the presence of this kind of basement below the Campanian Volcanic Province. This model has been called deep and hot intrusion zone by [5]. In addition, I state that the GLOSS of [19] are a mixing of the Archean upper crust and Hercynian basement as in rock cycle operating.

\section{Acknowledgements}

The $\mathrm{Pb}$ isotope data have been produced in the laboratories of USGS, Reston, VA, USA. Thanks to Robert Ayuso to have always hosted me during my PhD. Thanks to the patience of Benedetto De Vivo as tutor during my $\mathrm{PhD}$ (1994-1999). Thanks to Chris Hawkesworth for an early review.

\section{References}

[1] Paone, A. (2006) The Geochemical Evolution of Somma-Vesuvius Volcano. Mineralogy and Petrology, 87, 53-80. http://dx.doi.org/10.1007/s00710-005-0103-7

[2] Pappalardo, L., Piochi, M., D’Antonio, M., Civetta, L. and Petrini, R. (2002) Evidence for Multistage Magmatic Evolution during the Past 60 kyr at Campi Flegrei (Italy) Deduced from Sr, Nd, and Pb Isotope Data. Journal of Petroleum, 
43, 1415-1434. http://dx.doi.org/10.1093/petrology/43.8.1415

[3] Paone, A. (2004) Evidence of Crustal Contamination, Sediment, and Fluid Components in the Campanian Volcanic Rocks. Journal of Volcanology and Geothermal Research, 138, 1-26. http://dx.doi.org/10.1093/petrology/43.8.1415

[4] Paone, A. (2013) Petrogenesis of Trachyte and Rhyolite Magmatism on Ponza Island (Italy) and Its Relationship to the Campanian Magmatism. Journal of Volcanology and Geothermal Research, 267, 15-29. http://dx.doi.org/10.1016/j.jvolgeores.2013.09.008

[5] Annen, C. and Sparks, R.S.J. (2002) Effects of Repetitive Emplacement of Basaltic Intrusions on Thermal Evolution and Melt Generation in the Deep Crust. Earth Planet. Science Letters, 203, 937-955. http://dx.doi.org/10.1016/S0012-821X(02)00929-9

[6] Annen, C., Blundy, J.D. and Sparks, R.S.J. (2006) The Genesis of Intermediate and Silicic Magmasin Deep Crustal Hot Zones. Journal of Petrology, 47, 505-539. http://dx.doi.org/10.1093/petrology/egi084

[7] D’Antonio, M., Civetta, L. and Di Girolamo, P. (1999) Mantle Source Heterogeneity in the Campanian Region (South Italy) as Inferred from Geochemical and Isotopic Features of Mafic Volcanic Rocks with Shoshonitic Affinity. Mineralogy and Petrology, 67, 163-192. http://dx.doi.org/10.1007/BF01161520

[8] Scrocca, D., Doglioni, C. and Innocenti, F. (2003) Constraints for an Interpretation of the Italian Geodynamics: A Review. Memorie descrittive della carta geologica d'Italia Servizio geologico d'Italia, 62, 15-46.

[9] Doe, B.R. (1970) Lead Isotopes. Minerals, Rocks and Inorganic Materials. Monograph Series of Theoretical and Experimental Studies 3. Springer, Berlin, 143.

[10] Faure, G. and Mensing, T.M. (2004) Isotopes: Principles and Applications. John Wiley and Sons, Hoboken, 897.

[11] Dickin, A.P. (2005) Radiogenic Isotope Geology. 2nd Edition, Cambridge University Press, Cambridge, 509. http://dx.doi.org/10.1017/CBO9781139165150

[12] Picarelli, L., Evangelista, A., Rolandi, G., Paone, A., Nicotera, M., Olivares, L., Scotto di Santolo, A., Lampitiello, S. and Rolandi, M. (2006) Mechanical Properties and Behaviour of Pyroclastic Soils in Campania Region. Proceedings of the 2nd International Workshop on Characterisation and Engineering Properties of Natural Soils, Singapore, 29 November-1 December 2006, 2331-2384.

[13] De Vivo, B., Rolandi, G., Gans, P.B., Calvert, A., Bohrson, B.A., Spera, F.J. and Belkin, H.E. (2001) New Constraints on the Pyroclastic Eruptive History of the Campanian Volcanic Plain (Italy). Mineralogy and Petrology, 73, 47-65. http://dx.doi.org/10.1007/s007100170010

[14] De Vivo, B., Torok, K., Ayuso, R.A., Lima, A. and Lirer, L. (1995) Fluid Inclusion Evidence for Magmatic Silicate/Saline/ $\mathrm{CO}_{2}$ Immiscibility and Geochemistry of Alkaline Xenoliths from Ventotene Island, Italy. Geochimica et Cosmochimica Acta, 59, 2941-2953. http://dx.doi.org/10.1016/0016-7037(95)00186-7

[15] Ito, E., White, W.M. and Gopel, C. (1987) The O, Sr, Nd and Pb Isotope Geochemistry of MORB. Chemical Geology, 62, 157-176. http://dx.doi.org/10.1016/0009-2541(87)90083-0

[16] Rollinsson, H.G. (1993) Using Geochemical Data: Evaluation, Presentation, Interpretation. Pearson Education Limited, Harlow, 380.

[17] Rottura, A., Del Moro, A., Pinarelli, L., Petrini, R., Peccerillo, A., Caggianelli, G.M. and Piccarretta, G. (1991) Relationships between Intermediate and Acidic Rocks in Orogenic Granitoid Suites: Petrological, Geochemical and Isotopic (Sr, Nd, Pb) Data from Capo Vaticano (Southern Calabria, Italy). Chemical Geology, 92, 153-176. http://dx.doi.org/10.1016/0009-2541(91)90054-U

[18] Ayuso, R.A., Messina, A., De Vivo, B., Russo, S., Woodruff, L.G., Sutter, J.F. and Belkin, H.E. (1994) Geochemistry and Argon Thermochronology of the Variscan Sila Batholith, Southern Italy: Source Rocks and Magma Evolution. Contributions to Mineralogy and Petrology, 117, 87-109. http://dx.doi.org/10.1007/BF00307732

[19] Plank, T. and Langmuir, C.H. (1998) The Composition of Subducting Sediment and Its Consequences for the Crust and Mantle. Chemical Geology, 145, 325-394. http://dx.doi.org/10.1016/S0009-2541(97)00150-2

[20] Paone, A. (2013) A Review of Carbonatite Occurrences in Italy and Evaluation of Origins. Open Journal of Geology, 3, 66-82. http://dx.doi.org/10.4236/ojg.2013.32011 
Scientific Research Publishing (SCIRP) is one of the largest Open Access journal publishers. It is currently publishing more than 200 open access, online, peer-reviewed journals covering a wide range of academic disciplines. SCIRP serves the worldwide academic communities and contributes to the progress and application of science with its publication.

Other selected journals from SCIRP are listed as below. Submit your manuscript to us via either submit@scirp.org or Online Submission Portal.
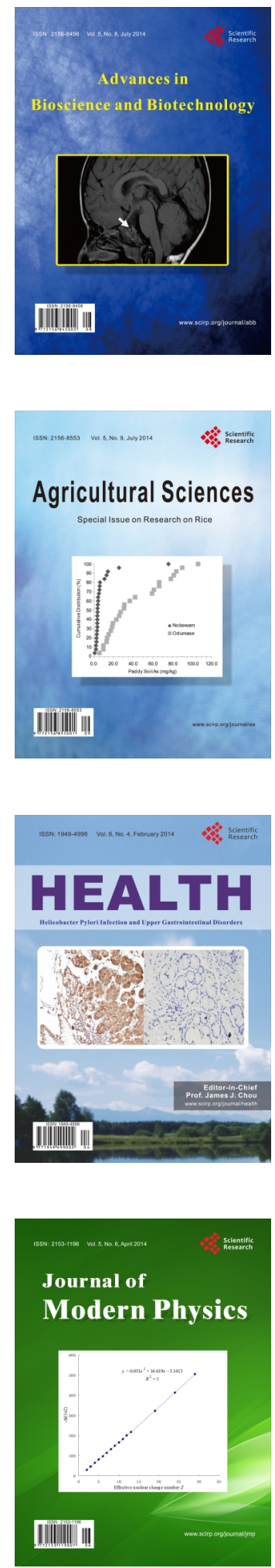
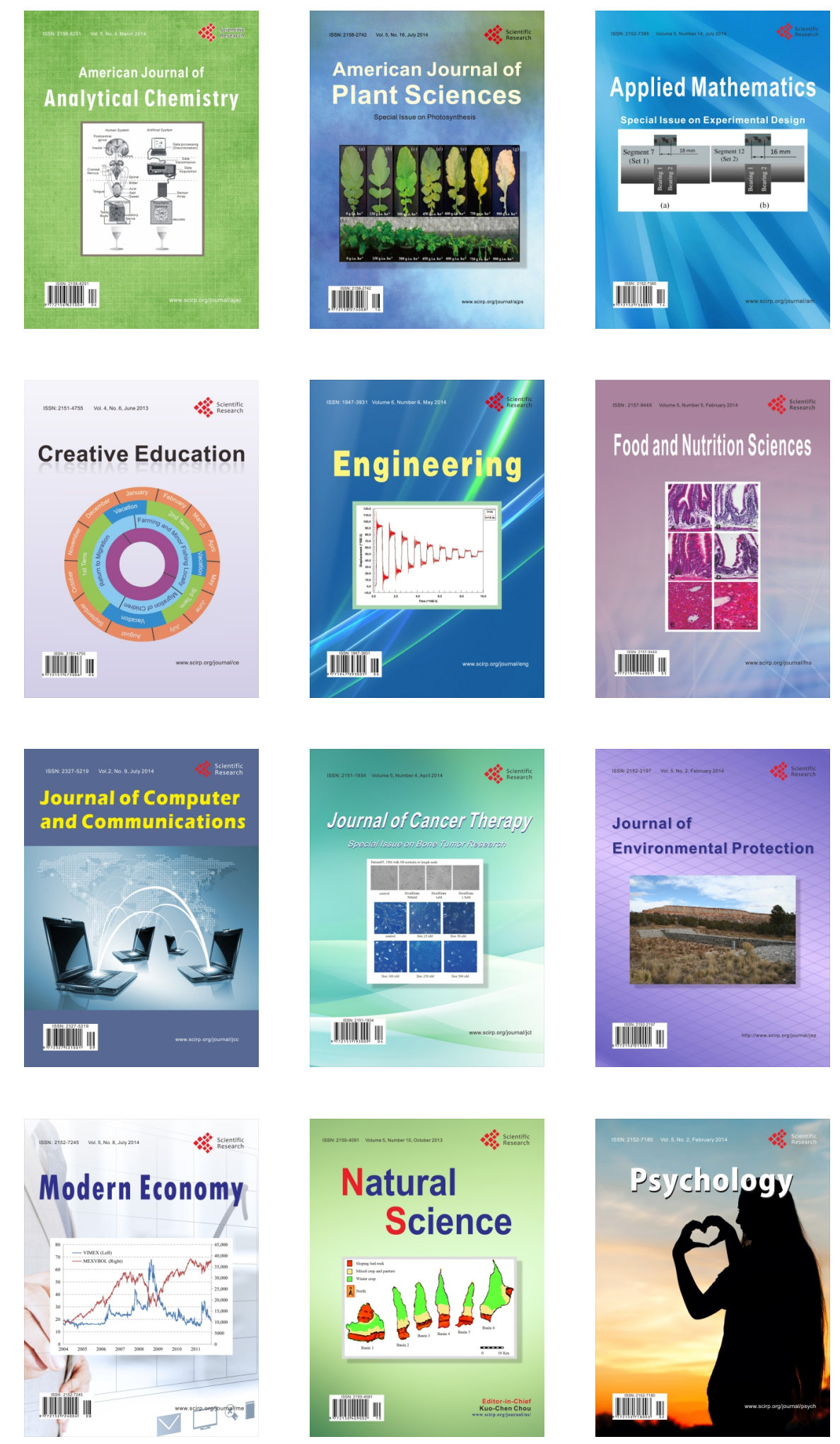